\title{
Induction of apoptosis by piceatannol in human leukemic U937 cells through down-regulation of Bcl-2 and activation of caspases
}

\author{
YEONG HOON KIM ${ }^{1,2 *}$, CHEOL PARK ${ }^{3,4}$, JEONG OK LEE $^{1}$, GI YOUNG KIM ${ }^{5}$, \\ WON HO LEE ${ }^{3}$, YUNG HYUN CHOI ${ }^{4,6}$ and CHUNG HO RYU ${ }^{1}$
}

\begin{abstract}
${ }^{1}$ Division of Applied Life Science, Institute of Agriculture and Life Science, Gyeongsang National University, Jinju 660-701; ${ }^{2}$ Gyeongsangnam-do Provincial Government Public Health and Environmental Research Institute, Chungwon 641-821; ${ }^{3}$ Department of Biology, Pusan National University, Busan 609-735; ${ }^{4}$ Department of Biochemistry, Dongeui University College of Oriental Medicine, Busan 614-052; ${ }^{5}$ Faculty of Applied Marine Science,

Cheju National University, Jeju 690-756; ${ }^{6}$ Department of Biomaterial Control (BK21 program),

Dongeui University Graduate School, Busan 614-052, Korea
\end{abstract}

Received June 27, 2007; Accepted October 15, 2007

\begin{abstract}
Piceatannol is a polyphenol that is found in abundant quantities in grapes and wine. Although recent experimental data revealed the proapoptotic potency of piceatannol, the molecular mechanisms underlying the anti-leukemic activity have not yet been studied in detail. This study examined the effects of piceatannol on the growth of the human leukemia cell line U937. The results showed that piceatannol inhibits the viability of U937 cells by inducing apoptosis, as evidenced by the formation of apoptotic bodies, DNA fragmentation and the accumulation of the sub-G1 phase. RT-PCR and immunoblotting data showed that treating the cells with piceatannol caused the down-regulation of anti-apoptotic Bcl-2 and cIAP-2 expression. Piceatannol-induced apoptosis was also associated with the proteolytic activation of caspase-3, and the degradation/cleavage of poly (ADP-ribose) polymerase protein. z-DEVD-fmk, a caspase-3-specific inhibitor, blocked the activation of caspase- 3 and increased the survival of the piceatannol-treated U937 cells, suggesting that caspase-3 activation is essential for piceatannol-induced apoptosis.
\end{abstract}

Correspondence to: Dr Chung Ho Ryu, Division of Applied Life Science, Institute of Agriculture and Life Science, Gyeongsang National University, Jinju 660-701, Korea

E-mail: ryu@gnu.ac.kr

Dr Yung Hyun Choi, Department of Biochemistry, Dongeui University College of Oriental Medicine, Busan 614-052, Korea

E-mail: choiyh@deu.ac.kr

*Contributed equally

Key words: piceatannol, apoptosis, Bcl-2, caspase-3

\section{Introduction}

Apoptosis, a programmed cell death, is an active form of cell death that plays a crucial role in the normal development and differentiation of multicellular organisms. It is characterized by a highly stereotypical series of morphological and biological changes, such as cytoplasmic shrinkage, blebbing of the plasma membrane, chromatin condensation and DNA degradation $(1,2)$. Apoptosis is a fundamental cellular activity that is essential for maintaining the physiological balance of an organism. It is involved in the immune defense machinery and plays an important role as a protective mechanism against carcinogenesis by eliminating damaged cells or the abnormal proliferation of cells (3). Apoptosis can be initiated by an extrinsic and an intrinsic pathway. Depending on the nature of a substance and the type of cells, programmed cell death may take place via either pathway. In the former case, plasma membrane death receptors are involved. The apoptosis signal is provided by the interaction between the ligand and death receptor. Changes in the mitochondrial integrity by a broad range of physical and chemical stimuli however, can trigger the intrinsic pathway of apoptosis $(4,5)$. Besides the morphological and biological changes, a lot of other biomarkers and events could be used for the precise determination of the type of apoptosis.

Piceatannol (3,3',4,5'-tetrahydroxy-trans-stilbene), a derivative of resveratrol, is a naturally occurring hydroxystilbene present in the skins of grapes, rhubarb, and sugar cane $(6,7)$. Piceatannol was first isolated from the seeds of Euphorbia lagascae (8) and preliminary data suggest that resveratrol is metabolized to piceatannol via cytochrome P4501B1 (9). This compound has been reported to be a protein-tyrosine kinase inhibitor with immunosuppressive activity, which could play an important role in preventing graft rejection (10-12). Additionally, piceatannol possesses antiinflammatory properties, suppressing the activation of the nuclear transcription factor nuclear factor $\kappa \mathrm{B}(\mathrm{NF}-\kappa \mathrm{B})$ through 
the inhibition of the inhibitor of $N F-\kappa B$ kinase and $\mathrm{p} 65$ phosphorylation (13-15). This compound was also demonstrated to prevent interferon- $\alpha$-induced inhibition of signal transducer and activator of transcription 3 (STAT3) and STAT3 phosphorylation in B and T lymphocytes (16-18). These results indicate that piceatannol abrogates proinflammatory responses by modifying multiple cellular targets. More recent studies have suggested that piceatannol inhibits the proliferation of cancer cells in culture by causing cell cycle arrest and apoptosis (17-22). Whether piceatannol acts as a potent chemopreventive agent for other types of tumors remains to be determined. This study examined the antiproliferative activity of piceatannol along with its effect on the apoptosis of human monocytic leukemic U937 cells. Furthermore, the levels of several important genes that are strongly associated with the signal transduction pathway of apoptosis were assayed to establish the anti-cancer mechanism of piceatannol as a potential chemopreventive agent.

\section{Materials and methods}

Cell culture and viability assay. The human leukemia cell line U937 was obtained from the American Type Culture Collection (Rockville, MD), and cultured in RPMI-1640 medium supplemented with $10 \%$ heat-inactivated fetal bovine serum (FBS), $2 \mathrm{mM}$ glutamine, $100 \mathrm{U} / \mathrm{ml}$ penicillin, and $100 \mu \mathrm{g} / \mathrm{ml}$ streptomycin (Gibco BRL, Gaithersburg, MD) at $37^{\circ} \mathrm{C}$ and $5 \% \mathrm{CO}_{2}$. Piceatannol was purchased from Sigma Chemical Co. (St. Louis, MO). A 20 mM concentration stock solution was made by dissolving piceatannol in dimethyl sulfoxide (DMSO). The solution was stored in aliquots at $-20^{\circ} \mathrm{C}$. For the viability study, the cells were cultured in the presence or absence of various piceatannol concentrations for $48 \mathrm{~h}$. The cells were trypsinized and washed with phosphate-buffered saline (PBS), and the viable cells were scored using a hemocytometer with trypan blue exclusion.

Nuclear staining with DAPI. The cells were washed with PBS and fixed with $3.7 \%$ paraformaldehyde in PBS for $10 \mathrm{~min}$ at room temperature. The fixed cells were washed with PBS, and stained with a 4,6-diamidino-2-phenylindole (DAPI, Sigma) solution for $10 \mathrm{~min}$ at room temperature. The cells were then washed twice with PBS and analyzed by fluorescence microscopy (Carl Zeiss, Germany).

Agarose gel electrophoresis for DNA fragmentation assay. After the piceatannol treatment, the cells were lysed in a buffer containing $10 \mathrm{mM}$ Tris- $\mathrm{HCl} \mathrm{pH} 7.4,150 \mathrm{mM} \mathrm{NaCl}, 5 \mathrm{mM}$ EDTA and $0.5 \%$ Triton X-100 for $30 \mathrm{~min}$ on ice. The lysates were vortexed and cleared by centrifugation at $10,000 \mathrm{x} \mathrm{g}$ for $20 \mathrm{~min}$. The DNA in the supernatant was extracted using a 25:24:1 (v/v/v) equal volume of neutral phenol:chloroform: isoamyl alcohol (Sigma) and analyzed electrophoretically on $1.2 \%$ agarose gels containing $0.1 \mu \mathrm{g} / \mathrm{ml}$ ethidium bromide (EtBr, Sigma).

Flow cytometry analysis for measurement of sub-G1 phase. The DNA content of the cells was measured using a DNA staining kit (CycleTest $^{\mathrm{TM}}$ plus kit, Becton Dickinson, San Jose, $\mathrm{CA}$ ). Propidium iodide (PI)-stained nuclear fractions were obtained using the instructions provided in the kit. Flow cytometric analyses were carried out using a flow cytometer (FACsCalibur, Becton Dikinson) and CellQuest software was used to determine the relative DNA content based on the presence of a red fluorescence.

$R N A$ extraction and reverse transcription- $P C R$. The total RNA was prepared using an RNeasy kit (Qiagen, La Jolla, CA) and primed with random hexamers to synthesize the complementary DNA using AMV reverse transcriptase (Amersham Corp., Arlington Heights, IL) according to the manufacturer's instructions. A polymerase chain reaction (PCR) was carried out using a Mastercycler (Eppendorf, Hamburg, Germany) with the primers shown in Table I. The following conditions were used for the PCR reactions: $1 \mathrm{x}\left(94^{\circ} \mathrm{C}\right.$ for $\left.3 \mathrm{~min}\right) ; 35 \mathrm{x}$ $\left(94^{\circ} \mathrm{C}\right.$ for $45 \mathrm{sec} ; 58^{\circ} \mathrm{C}$ for $45 \mathrm{sec}$; and $72^{\circ} \mathrm{C}$ for $\left.1 \mathrm{~min}\right)$ and $1 \mathrm{x}$ $\left(72^{\circ} \mathrm{C}\right.$ for $\left.10 \mathrm{~min}\right)$. The amplification products obtained by PCR were separated electrophoretically on a $1 \%$ agarose gel and visualized by EtBr staining.

Gel electrophoresis and Western blot analysis. The cells were harvested, lysed, and the protein concentrations were quantified using a BioRad protein assay (BioRad Lab., Hercules, CA), according to the procedure reported by the manufacturer. For Western blot analysis, an equal amount of protein was subjected to electrophoresis on SDSpolyacrylamide gel and transferred to a nitrocellulose membrane (Schleicher \& Schuell, Keene, NH) by electroblotting. The blots were probed with the desired antibodies for $1 \mathrm{~h}$, incubated with the diluted enzyme-linked secondary antibody and visualized by enhanced chemiluminescence (ECL) according to the recommended procedure (Amersham). The primary antibodies were purchased from Santa Cruz Biotechnology Inc. (Santa Cruz, CA) and Calbiochem (Cambridge, MA). The peroxidase-labeled donkey anti-rabbit immunoglobulin and peroxidase-labeled sheep anti-mouse immunoglobulin were purchased from Amersham.

Assay of caspase-3, -8 and -9 activity. The enzymatic activity of the caspases induced by piceatannol was assayed using a colorimetric assay kit according to the manufacturer's protocol (R\&D Systems, Minneapolis, MN). Briefly, the cells were lysed in a lysis buffer for $30 \mathrm{~min}$ on an ice bath. The lysed cells were centrifuged at $12,000 \mathrm{x}$ g for $10 \mathrm{~min}$, and $100 \mu \mathrm{g}$ of the protein was incubated with $50 \mu \mathrm{l}$ of a reaction buffer and $5 \mu 1$ of the colorimetric tetrapeptides, Asp-Glu-Val-Asp (DEVD)-p-nitroaniline (pNA) for caspase-3, Ile-Glu-Thr-Asp (IETD)-pNA for caspase-8 and Leu-Glu-HisAsp (LEHD)-pNA for caspase-9, respectively, at $37^{\circ} \mathrm{C}$ for $2 \mathrm{~h}$. The optical density of the reaction mixture was quantified spectrophotometrically at a wavelength of $405 \mathrm{~nm}$.

\section{Results}

Piceatannol-inhibited cell viability and induced apoptosis in U937. The piceatannol-induced cell cytotoxicity was determined from the effects of different piceatannol concentrations on the U937 cell viability. The data showed that a treatment with piceatannol for $48 \mathrm{~h}$ decreased the 
Table I. Gene-specific primers for RT-PCR

\begin{tabular}{|c|c|c|}
\hline Name & & Sequence of primers \\
\hline \multirow[t]{2}{*}{ Fas } & Sense & 5'-TCT-AAC-TTG-GGG-TGG-CTT-TGT-CTT-C-3' \\
\hline & Antisense & 5'-GTG-TCA-TAC-GCT-TTC-TTT-CCA-T-3' \\
\hline \multirow[t]{2}{*}{ FasL } & Sense & 5'-GGA-TTG-GGC-CTG-GGG-ATG-TTT-CA-3' \\
\hline & Antisense & 5'-AGC-CCA-GTT-TCA-TTG-ATC-ACA-AGG-3' \\
\hline \multirow[t]{2}{*}{ Bax } & Sense & 5'-ATG-GAC-GGG-TCC-GGG-GAG-3' \\
\hline & Antisense & 5'-TGG-AAG-AAG-ATG-GGC-TGA-3' \\
\hline \multirow[t]{2}{*}{ Bcl-2 } & Sense & 5'-CAG-CTG-CAC-CTG-ACG-3' \\
\hline & Antisense & 5'-GCT-GGG-TAG-GTG-CAT-3' \\
\hline \multirow[t]{2}{*}{ Bcl-xL } & Sense & 5'-CAG CTG CAC CTG ACG-3' \\
\hline & Antisense & 5'-GCT GGG TAG GTG CAT-3' \\
\hline \multirow[t]{2}{*}{ XIAP } & Sense & 5'-GAA-GAC-CCT-TGG-GAA-CAA-CA-3' \\
\hline & Antisense & 5'-CGC-CTT-AGC-TGC-TCT-CTT-CAG-T-3' \\
\hline \multirow[t]{2}{*}{ cIAP-1 } & Sense & 5'-TGA-GCA-TGC-AGA-CAC-ATG-C-3' \\
\hline & Antisense & 5'-TGA-CGG-ATG-AAC-TCC-TGT-CC-3' \\
\hline \multirow[t]{2}{*}{ cIAP-2 } & Sense & 5'-CAG-AAT-TGG-CAA-GAG-CTG-G-3' \\
\hline & Antisense & 5'-CAC-TTG-CAA-GCT-GCT-CAG-G-3' \\
\hline \multirow[t]{2}{*}{ GAPDH } & Sense & 5'-CGG-AGT-CAA-CGG-ATT-TGG-TCG-TAT-3' \\
\hline & Antisense & 5'-AGC-CTT-CTC-CAT-GGT-GGT-GAA-GAC-3' \\
\hline
\end{tabular}

A)

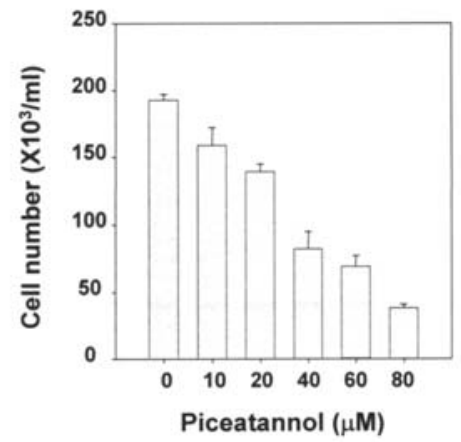

B)
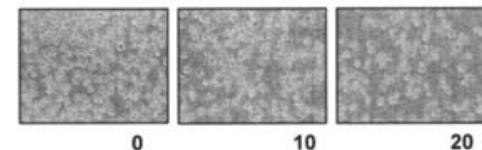

0

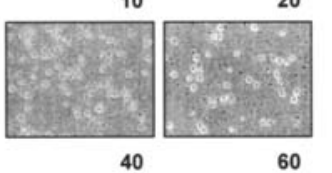

Piceatannol $(\mu \mathrm{M})$

Figure 1. Inhibition of the cell viability by piceatannol in U937 human leukemic cells. (A) The cells were plated at $1 \times 10^{3}$ cells per $60-\mathrm{mm}$ plate, and incubated for $24 \mathrm{~h}$. The cells were treated with different concentrations of piceatannol for $48 \mathrm{~h}$, according to the measurements of the cell viability by hemocytometer counts of trypan blue-excluding cells. The data are reported as mean \pm S.D. of three independent experiments. (B) The cells grown under the same conditions as (A) were examined under a light microscopy. The data are a representative example for duplicate tests. Magnification, x200. viability of U937 cells in a concentration-dependent manner (Fig. 1). Further experiments were carried out to determine if this inhibitory effect of piceatannol on cell viability is the result of apoptotic cell death. By morphological analysis with DAPI staining, the nuclei with chromatin condensation and the formation of apoptotic bodies were observed in the cells cultured with piceatannol in a concentration-dependent manner, but very few were observed in the control culture (Fig. 2A). The following experiments were carried out to determine if piceatannol induces DNA fragmentation in U937 cells. Piceatannol induced the progressive accumulation of fragmented DNA, which appeared as a typical ladder pattern of DNA fragmentation due to internucleosomal cleavage associated with apoptosis, in a concentration-dependent manner (Fig. 2B). The degree of apoptosis was determined by analyzing the amount of sub-G1 DNA in the U937 cells treated with piceatannol using flow cytometry. As shown in Fig. 2C, the addition of piceatannol to the U937 cells resulted in the increased accumulation of cells in the sub-G1 phase in a similar manner to that observed with the piceatannol-induced loss of cell viability, formation of apoptotic bodies and accumulation of extra-nuclear fragmented DNA. This suggests that the cells can undergo apoptosis after exposing the U937 cells to piceatannol, and there is a good correlation between the extent of apoptosis and the inhibition of cell viability.

Effects of piceatannol on the expression of Fas/FasL, Bcl-2 and the IAP family. The expression levels in piceatannoltreated U937 cells were examined by RT-PCR and Western 
A)

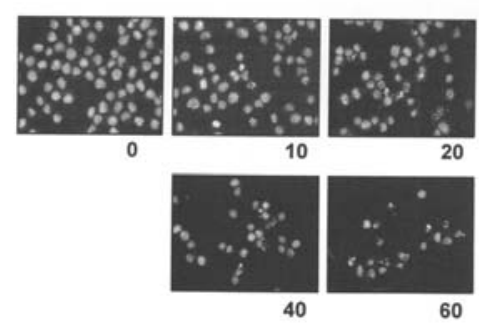

Piceatannol $(\mu \mathrm{M})$
B)

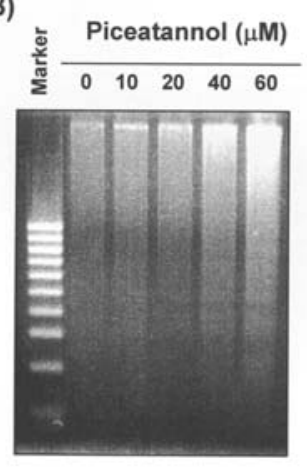

C)

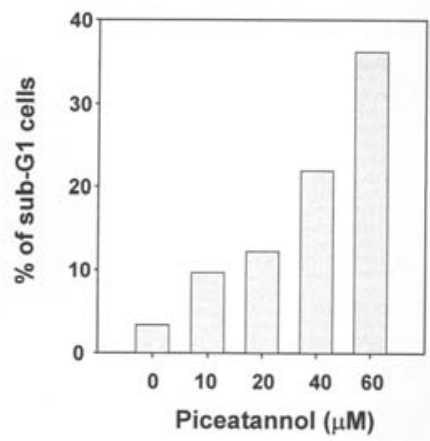

Figure 2. Induction of apoptosis by piceatannol in U937 human leukemic cells. (A) After treating the cells with piceatannol for 48 h, they were stained with DAPI. After 10 min incubation, the cells were washed with PBS and photographed with a fluorescence microscope using a blue filter. Magnification, x400. (B) For the analysis of DNA fragmentation, the U937 cells were treated with piceatannol for $48 \mathrm{~h}$. The genomic DNA was extracted, and analyzed on a $1.2 \%$ agarose gel. (C) To quantify the degree of apoptosis induced by piceatannol, the cells were evaluated for the sub-G1 DNA content using a flow cytometer. Each point represents the average of two independent experiments.

A)

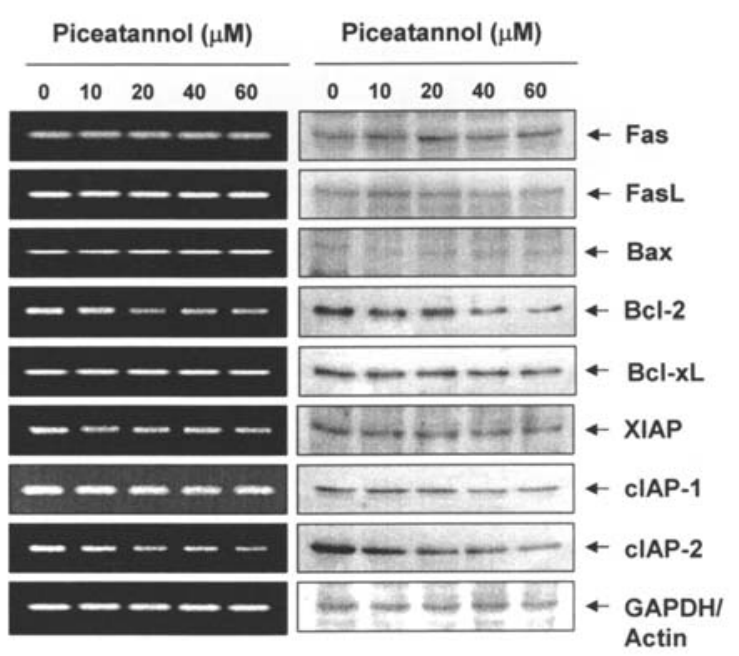

Figure 3. The effects of piceatannol on the expression of Bcl-2, Fas/FasL and the IAP family in U937 cells. (A) The cells were incubated with different piceatannol concentrations for $48 \mathrm{~h}$, and the total RNA was isolated and reverse-transcribed. The resulting cDNAs were subjected to PCR with the indicated primers and the reaction products were subjected to electrophoresis in a $1 \%$ agarose gel and visualized by EtBr staining. GAPDH was used as the internal control. (B) The cells grown under the same conditions as (A) were lysed and equal amounts of proteins were then separated by SDS-polyacrylamide gels and transferred to nitrocellulose membranes. The membranes were probed with the indicated antibodies and detected by ECL. To confirm equal loading, the blot was stripped of the bound antibody and reprobed with the anti-actin antibody.

blotting in order to determine if piceatannol induces U937 cell death through a change in the expression of the Fas/FasL, Bcl-2 or IAP family members. As shown in Fig. 3, the addition of piceatannol to U937 cells did not cause significant changes in the expression of Fas, FasL, Bax, Bcl$\mathrm{xL}$, XIAP and cIAP-1. However, piceatannol inhibited the concentration-dependent decrease in the expression levels of the Bcl-2 and cIAP-2 mRNA and proteins. The downregulation of both Bcl-2 and cIAP-2 by piceatannol occurred in a similar concentration-dependent manner to that observed with the inhibition of cell viability and induction of apoptosis.
These results suggest that piceatannol specifically downregulates the expression levels of Bcl-2 and cIAP-2 in U937 cells.

Activation of caspase-3 and -9, and degradation of PARP by piceatannol. The expression levels and activities of caspase$3,-8$ and -9 in U937 cells that had been exposed to various concentrations of piceatannol for $48 \mathrm{~h}$ were measured in order to determine if piceatannol-induced apoptosis is associated with the activation of caspases. As shown in Fig. 3A, treatment with piceatannol decreased the levels of procaspase- 3 and caspase- 9 expression. However, the caspase- 8 expression levels in the piceatannol-treated cells were relatively unaffected. The activation of caspase often leads to the proteolytic cleavage of several target proteins such as poly-(ADP-ribose) polymerase (PARP), which is a downstream target of activated caspase-3 (23). Subsequent Western blot analyses showed the progressive proteolytic cleavage of PARP in U937 cells after the treatment with piceatannol. The proteolytic activity of caspases was determined using an in vitro assay with a colorimetric assay kit. Treatment with piceatannol increased the caspase-3 and -9 activities ( $~ 5.1$-fold and 3.5-fold after $48 \mathrm{~h}$ exposure to $60 \mu \mathrm{M}$ piceatannol, respectively) in a concentrationdependent manner but caspase- 8 was not significantly activated in the piceatannol-treated cells (Fig. 3B).

Inhibition of piceatannol-induced apoptosis by caspase-3 inhibitor. In order to show that the activation of caspase- 3 is a key step in the piceatannol-induced apoptotic pathway, the U937 cells were pretreated with z-DEVD-fmk $(50 \mu \mathrm{M})$, a cell-permeable caspase- 3 inhibitor, for $1 \mathrm{~h}$, followed by a treatment with $60 \mu \mathrm{M}$ piceatannol for $48 \mathrm{~h}$. The blockade of the caspase-3 activity by a pre-treatment of the cells with zDEVD-fmk prevented the piceatannol-induced chromatin condensation and increase in the sub-G1 population (Fig. 5B and D). Furthermore, z-DEVD-fmk significantly blocked not only caspase-3 cleavage but also PARP degradation (Fig. 5C) in the piceatannol-treated U937 cells. These results clearly show that piceatannol-induced apoptosis is associated with caspase-3 activation. 
A)

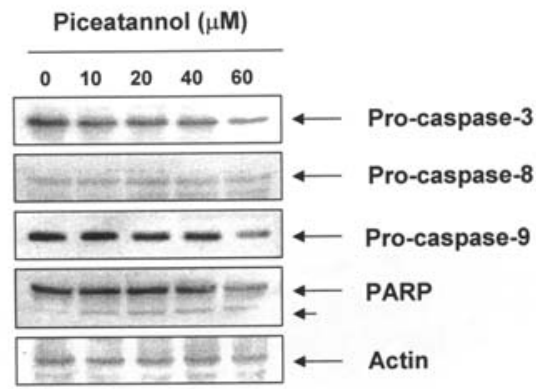

B)

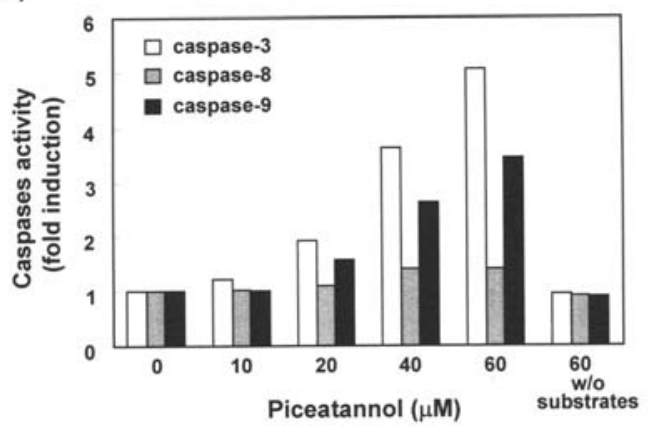

Figure 4. Activation of caspase-3 and -9 , and the degradation of the PARP protein by piceatannol in U937 cells. (A) After $48 \mathrm{~h}$ incubation with piceatannol, the cells were lysed and the cellular proteins were separated by SDS-polyacrylamide gels and transferred onto nitrocellulose membranes. The membranes were probed with the anti-caspase-3, anti-caspase- 8 , anticaspase-9 and anti-PARP antibodies. The proteins were visualized using an ECL detection system. Actin was used as the internal control. (B) The cell lysates from the cells treated with piceatannol for $48 \mathrm{~h}$ were assayed for in vitro caspase-3, -8 and -9 activity using DEVD-pNA, IETD-pNA and LEHD-pNA respectively, as substrates. The released fluorescent products were measured. The data represent the average of two independent experiments.

\section{Discussion}

Many biological effects of piceatannol, including antiinflammatory, immune-modulating, and chemopreventive activities have been described, which suggests that piceatannol might be an effective natural component in cancer chemoprevention. Several studies have reported that piceatannol treatment caused the accumulation of cells in the $\mathrm{G} 2 / \mathrm{M}$ phase of the cell cycle and induced apoptosis, suggesting that the growth inhibitory effect of piceatannol is the result of a block during the G2/M phase and that such cells do not enter the G1 phase (17-22). While the cell killing mechanism of piceatannol has been suggested, the precise cell death mechanism of piceatannol is not completely understood. Therefore, this study investigated the effects of piceatannol on the growth of the human leukemic U937 cell line in order to examine the mechanisms of its anti-proliferative pathway. Initially, it was found that piceatannol inhibits cell viability and induces the appearance of a sub-G1 cell population, which is considered to be a marker of cell death by apoptosis (24). Consistent with this observation, it was shown that piceatannol induces the dose-dependent apoptotic body formation and DNA fragmentation, which are end-stage apoptotic events (Fig. 2).

Apoptosis is a tightly regulated process under the control of several signaling pathways. One of the major genes that regulate apoptosis is the Bcl-2 family. In mammals, members
A)

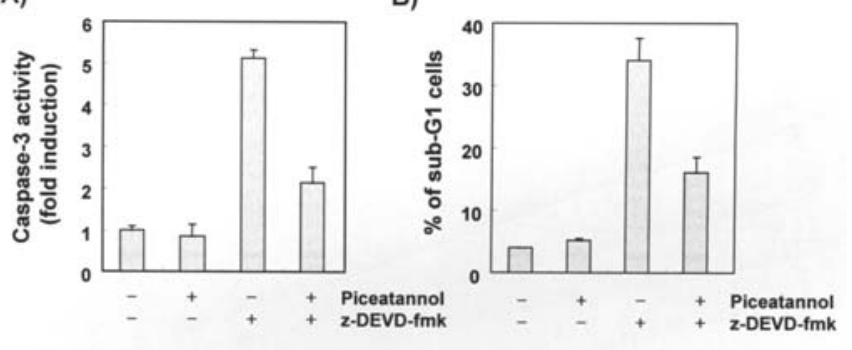

C)

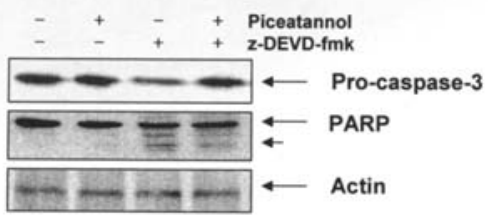

D)

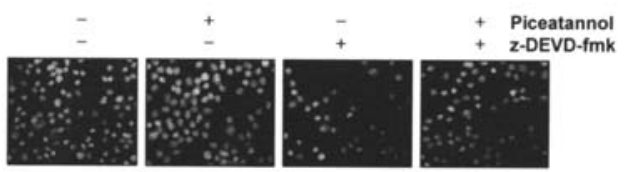

Figure 5. Inhibition of piceatannol-induced apoptosis by the caspase-3 inhibitor in U937 cells. (A) The cells were treated with z-DEVD-fmk $(50 \mu \mathrm{M})$ for $1 \mathrm{~h}$ before a challenge with $60 \mu \mathrm{M}$ piceatannol for $48 \mathrm{~h}$. The cell lysates were made and used to measure the caspase- 3 activity using DEVD-pNA, a caspase- 3 substrate. The data are reported as the mean values from three independent experiments and the bars represent the standard deviations. (B) The cells grown under the same conditions as (A) were evaluated for the sub-G1 DNA content using a flow cytometer. The data are reported as mean \pm S.D. of three independent experiments. (C) Equal amounts of proteins extracted from cells grown under the same conditions as (A) were separated by SDS-polyacrylamide gels and transferred onto nitrocellulose membranes. The membranes were probed with the indicated antibodies. The proteins were visualized using an ECL detection system. Actin was used as the internal control. (D) U937 cells were pretreated for $1 \mathrm{~h}$ with or without $\mathrm{z}$-DEVD-fmk, and then with piceatannol for an additional $48 \mathrm{~h}$. The cells were stained with DAPI for $10 \mathrm{~min}$ and photographed with a fluorescence microscope using a blue filter. Magnification, $\mathrm{x} 400$.

of the Bcl-2 family can be divided into two subfamilies; the anti-apoptotic protein family such as Bcl-2 and Bcl-xL, and the pro-apoptotic protein family such as Bax and Bak. It has been reported that $\mathrm{Bcl}-2$ members protect against multiple signals that lead to cell death, whereas Bax members induce apoptosis, indicating that the Bcl-2 family regulates a common cell death pathway and functions at a point where various signals converge. The most well-known apoptogenic factor released from the permeabilized mitochondria is the respiratory component cytochrome $c$, which recruits the apoptosis protease activating factor (Apaf-1) and procaspase-9 to form apoptosome. Caspase- 9 is then activated, and orchestrates caspase- 3 and other effector molecules for cell death $(25,26)$. Bcl-2 acts to inhibit cytochrome $c$ translocation from mitochodria to cytoplasm, thereby blocking the caspase activation step of the apoptotic process $(26,27)$. Thus, it has been suggested that the ratio between the level of pro-apoptotic Bax and that of the anti-apoptotic factor Bcl-2 determines whether a cell responds to an apoptotic signal. In the present study, there were no detectable changes in the levels of Bax and Bcl-xL in piceatannol-treated U937 cells, but the levels of Bcl-2 mRNA and protein expression were increased in a concentration- 
dependent manner, resulting in an increase in the ratio of Bax/Bcl-2 (Fig. 3). Further experiments showed that exposing U937 cells to piceatannol resulted in the proteolytic activation of caspase- 3 and caspase- 9 without activating caspase- 8 (Fig. 4). Activated caspases induce limited proteolysis in a number of cellular proteins, which are degraded as a result of apoptosis by the caspase family, and have been used as a marker of chemotherapy-induced apoptosis. This study examined whether or not PARP proteins, which are substrates of caspase-3, a main executioner of apoptosis (23), are cleaved in the cells treated with piceatannol. As expected, PARP proteins were clearly degraded in a dose-dependent manner, which correlated with the activation of capase-3 during apoptosis caused by the piceatannol treatment (Fig. 4). Under the same conditions, the caspase- 3 inhibitor z-DEVDfmk markedly prevented the piceatannol-induced apoptosis by blocking not only caspase-3 activation but also PARP cleavage (Figs. 4 and 5). This indicates that caspase-3 plays the role as a key molecule in piceatannol-induced apoptosis in U937 cells.

The IAP family have been reported to exert anti-apoptotic effects due to their function as direct inhibitors of activated caspases. Therefore, down-regulation of IAPs relieves the triggering block of proapoptotic signaling and the execution caspases, thus activating cell death $(28,29)$. The Fas/FasL system is also a key signaling transduction pathway of apoptosis in cells. Binding FasL to the Fas receptor leads to receptor oligomerization and the formation of a death-inducing signaling complex, followed by the activation of caspase-8, then further activating a series of caspase cascades resulting in apoptotic cell death $(30,31)$. Further studies have shown that exposure of U937 cells to piceatannol caused a downregulation of cIAP-2 expression, however, the levels of XIAP, cIAP-1, Fas and FasL expression remained unchanged in piceatannol-mediated U937 cellular apoptosis (Fig. 3). Therefore, the present data indicate that the apoptotic effects of piceatannol on U937 cells are associated with the activation of caspase through an increase in the ratio of $\mathrm{Bax} / \mathrm{Bcl}-2$ expression and the selective alteration of IAPs expression. Although further studies are needed, these results provide information on the possible mechanisms for the anticancer activity of piceatannol.

\section{Acknowledgements}

This work was supported by grant No. RTI04-03-07 from the Regional Technology Innovation Program of the Ministry of Commerce, Industry and Energy (MOCIE).

\section{References}

1. Walker PR and Sikorska M: New aspects of the mechanism of DNA fragmentation in apoptosis. Biochem Cell Biol 75: 287-299, 1997.

2. Robertson JD and Orrenius S: Molecular mechanisms of apoptosis induced by cytotoxic chemicals. Crit Rev Toxicol 30: 609-627, 2000.

3. Hengartner MO: The biochemistry of apoptosis. Nature 407: 770-776, 2000.

4. Ghobrial IM, Witzig TE and Adjei AA: Targeting apoptosis pathways in cancer therapy. CA Cancer J Clin 55: 178-194, 2005.

5. Takeda K, Stagg J, Yagita H, Okumura K and Smyth MJ: Targeting death-inducing receptors in cancer therapy. Oncogene 26: 3745-3757, 2007.
6. Bavaresco L, Fregoni C, Cantu E and Trevisan M: Stilbene compounds: from the grapevine to wine. Drugs Exp Clin Res 25: 57-63, 1999.

7. Roupe K, Teng XW, Fu X, Meadows GG and Davies NM: Determination of piceatannol in rat serum and liver microsomes: pharmacokinetics and phase I and II biotransformation. Biomed Chromatogr 18: 486-491, 2004.

8. Ferrigni NR, McLaughlin JL, Powell RG and Smith CR Jr: Use of potato disc and brine shrimp bioassays to detect activity and isolate piceatannol as the antileukemic principle from the seeds of Euphorbia lagascae. J Nat Prod 47: 347-352, 1984.

9. Potter GA, Patterson LH, Wanogho E, Perry PJ, Butler PC, Ijaz T, Ruparelia KC, Lamb JH, Farmer PB, Stanley LA and Burke MD: The cancer preventative agent resveratrol is converted to the anticancer agent piceatannol by the cytochrome P450 enzyme CYP1B1. Br J Cancer 86: 774-778, 2002.

10. Geahlen RL and McLaughlin JL: Piceatannol (3,4,3',5'tetrahydroxy-trans-stilbene) is a naturally occurring proteintyrosine kinase inhibitor. Biochem Biophys Res Commun 165: 241-245, 1989.

11. Fernandez LA, Torrealba J, Yagci G, Ishido N, Tsuchida M, Tae Kim H, Dong Y, Oberley T, Fechner J, Colburn MJ, Schultz J, Kanmaz T, Hu H, Knechtle SJ and Hamawy MM: Piceatannol in combination with low doses of cyclosporine A prolongs kidney allograft survival in a stringent rat transplantation model. Transplantation 74: 1609-1617, 2002.

12. Wong WS and Leong KP: Tyrosine kinase inhibitors: a new approach for asthma. Biochim Biophys Acta 1697: 53-69, 2004.

13. Ashikawa K, Majumdar S, Banerjee S, Bharti AC, Shishodia S and Aggarwal BB: Piceatannol inhibits TNF-induced NF- $\kappa B$ activation and $\mathrm{NF}-\kappa \mathrm{B}-$ mediated gene expression through suppression of IкB $\alpha$ kinase and $\mathrm{p} 65$ phosphorylation. J Immunol 169: 6490-6497, 2002.

14. Islam S, Hassan F, Mu MM, Ito H, Koide N, Mori I, Yoshida T and Yokochi T: Piceatannol prevents lipopolysaccharide (LPS)induced nitric oxide (NO) production and nuclear factor (NF)- $\mathrm{kB}$ activation by inhibiting IкB kinase (IKK). Microbiol Immunol 48: 729-736, 2004.

15. Jin CY, Moon DO, Lee KJ, Kim MO, Lee JD, Choi YH, Park YM and Kim GY: Piceatannol attenuates lipopolysaccharide-induced $\mathrm{NF}-\kappa \mathrm{B}$ activation and $\mathrm{NF}-\kappa \mathrm{B}$-related proinflammatory mediators in BV2 microglia. Pharmacol Res 54: 461-467, 2006.

16. Su L and David M: Distinct mechanisms of STAT phosphorylation via the interferon-alpha/beta receptor. Selective inhibition of STAT3 and STAT5 by piceatannol. J Biol Chem 275: 12661-12666, 2000.

17. Larrosa M, Tomas-Barberan FA and Espin JC: The grape and wine polyphenol piceatannol is a potent inducer of apoptosis in human SK-Mel-28 melanoma cells. Eur J Nutr 43: 275-284, 2004.

18. Kumari AL, Ali AM, Das S, Pardhasaradhi BV, Varalakshmi CH and Khar A: Role of STAT3 and NFKB signaling in the serum factor-induced apoptosis in AK-5 cells. Biochem Biophys Res Commun 336: 860-867, 2005.

19. Wieder T, Prokop A, Bagci B, Essmann F, Bernicke D, Schulze-Osthoff K, Dorken B, Schmalz HG, Daniel PT and Henze G: Piceatannol, a hydroxylated analog of the chemopreventive agent resveratrol, is a potent inducer of apoptosis in the lymphoma cell line BJAB and in primary, leukemic lymphoblasts. Leukemia 15: 1735-1742, 2001.

20. Larrosa M, Tomas-Barberan FA and Espin JC: Grape polyphenol resveratrol and the related molecule 4-hydroxystilbene induce growth inhibition, apoptosis, S-phase arrest, and upregulation of cyclins A, E, and B1 in human SK-Mel-28 melanoma cells. J Agric Food Chem 51: 4576-4584, 2003.

21. Barton BE, Karras JG, Murphy TF, Barton A and Huang HF: Signal transducer and activator of transcription 3 (STAT3) activation in prostate cancer: Direct STAT3 inhibition induces apoptosis in prostate cancer lines. Mol Cancer Ther 3: 11-20, 2004.

22. Ferreira MJ, Duarte N, Gyemant N, Radics R, Cherepnev G, Varga A and Molnar J: Interaction between doxorubicin and the resistance modifier stilbene on multidrug resistant mouse lymphoma and human breast cancer cells. Anticancer Res 26: 3541-3546, 2006.

23. Lazebnik YA, Kaufmann SH, Desnoyers S, Poirier GG and Earnshaw WC: Cleavage of poly (ADP-ribose) polymerase by a proteinase with properties like ICE. Nature 371: 346-347, 1994. 
24. Nicoletti I, Migliorati G, Pagliacci MC, Grignani F and Riccardi C: A rapid and simple method for measuring thymocyte apoptosis by propidium iodide staining and flow cytometry. $\mathrm{J}$ Immunol Methods 139: 271-279, 1991.

25. Rodriguez J and Lazebnik Y: Caspase- 9 and APAF-1 form an active holoenzyme. Genes 13: 3179-3184, 1999.

26. Philchenkov A, Zavelevich M, Kroczak TJ and Los M: Caspases and cancer: mechanisms of inactivation and new treatment modalities. Exp Oncol 26: 82-97, 2004.

27. Stennicke HR and Salvesen GS: Caspases - controlling intracellular signals by protease zymogen activation. Biochim Biophys Acta 1477: 299-306, 2000.
28. Roy N, Deveraux QL, Takahashi R, Salvesen GS and Reed JC: The c-IAP-1 and c-IAP-2 proteins are direct inhibitors of specific caspases. EMBO J 16: 6914-6925, 1997.

29. Deveraux QL and Reed JC: IAP family proteins-suppressors of apoptosis. Genes Dev 13: 239-252, 1999.

30. Nagata S and Golstein P: The Fas death factor. Science 267: 1449-1456, 1996.

31. Jiang C, Yang YF and Cheng SH: Fas ligand gene therapy for vascular intimal hyperplasia. Curr Gene Ther 4: 33-39, 2004. 